\title{
HST FOC and Broad Band Colors for Young and Intermediate Simple Stellar Populations
}

\section{E. Brocato}

Oss. Astr. Collurania, Via M. Maggini, I-64100 Teramo, Italy

V. Castellani

Dip. di Fisica, Univ. Pisa, Piazza Torricelli 2, I-56126 Pisa, Italy

G. Raimondo

Oss. Astr. Collurania, Via M. Maggini, I-64100 Teramo, Italy

M. Romaniello

STScI, 3700 San Martin Drive, Baltimore, MD 21218, USA

\begin{abstract}
.
We present theoretical HST and broad band colors from young and intermediate stellar populations with age ranging from $t=8 \mathrm{Myr}$ to $\mathrm{t}=5$ Gyr and three different metallicities $Z=0.02,0.006$ and 0.001 . We suggest the combined use of HST UV colors and IR colors (V-K in particular) to disentangle the metallicity-age effect in integrated colors of young stellar populations $(\mathrm{t} \leq 1 \mathrm{Gyr})$.
\end{abstract}

\section{Introduction}

The challenge of interpreting integrated spectra and/or colors of distant objects (galaxies and stellar clusters) in terms of stellar ages and chemical compositions was gathered approaching the problem of population synthesis both from a theoretical and an observational point of view.

The homogeneity of adopted library of stellar evolutionary tracks is the basic assumption which determines the predicted integrated properties of a Simple Stellar Population (SSP). To cover the main evolutionary phases expected, we will rely on the extensive set of stellar models (Teramo-Pisa-Frascati cooperation) presented for high (Brocato \& Castellani 1993), intermediate (Cassisi, Castellani \& Straniero 1994) and low mass (Straniero \& Chieffi 1991; Castellani, Chieffi \& Straniero 1992) stars.

\section{Discussion}

By using this homogeneous set of stellar evolutionary tracks as computed under canonical (no overshooting) assumptions, we computed theoretical HST and 

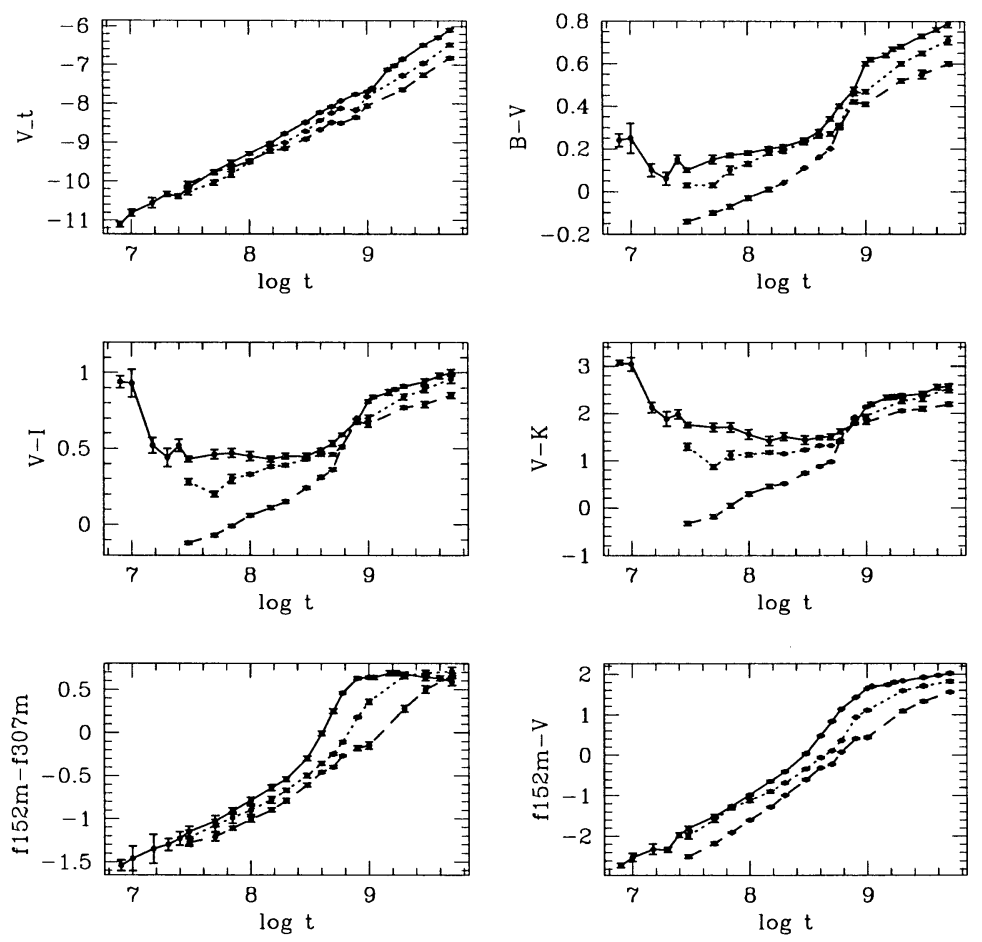

Figure 1. Time evolution of integrated $V$ magnitude and colors for three different assumption on metallicities: $\mathrm{Z}=0.02$ (solid lines), $\mathrm{Z}=$ 0.006 (dotted lines) and $\mathrm{Z}=0.001$ (dashed lines).

broad band colors from population synthesis models, covering the range of cluster ages from $\mathrm{t}=8 \mathrm{Myr}$ to $\mathrm{t}=5 \mathrm{Gyr}$ for three different metallicities $\mathrm{Z}=0.02$, 0.006 , and 0.001 (Fig. 1).

We find that for F152M-F307M $<0.5$ and F170M-F278M $<0.5$ (which means ages lower than $1 \mathrm{Gyr}$ ) the HST UV colors can still be used to infer reliable indication on the age of distant clusters. On these basis, we present a calibration of the HST UV two-colors (F152M-F307M, F170M-F278M) in terms of cluster ages for the three above quoted metallicities. Theoretical predictions for the time behavior of broad band colors $(U B V K)$ appear in good agreement with data for LMC stellar clusters with known age. We suggest that the combined use of HST UV colors and near-IR colors ( $V-K$ in particular) should disentangle the metallicity-age effect in integrated colors of young stellar populations $(\mathrm{t} \leq$ $1 \mathrm{Gyr}$ ). For this reason the NICMOS camera on board HST, particularly its F222M filter, is expected to be a powerful tool in interpreting the integrated colors of distant objects in term of age and metallicity.

MC stellar clusters represent a very fundamental test to check the reliability of population synthesis models. Unfortunately, age and metallicity determinations of single clusters available up to now are based, with few exceptions, on indirect calibrations. Thus, an extensive observational work with the goal of de- 
riving accurate and precise CMDs for a large sample of $\mathrm{MC}$ stellar cluster would be of invaluable help to check the reliability of population synthesis results.

\section{References}

Brocato E., \& Castellani V. 1993, ApJ, 410, 99

Cassisi S., Castellani V., \& Straniero O. 1994, A\&A, 282, 753

Castellani V.,Chieffi A., \& Straniero O. 1992, ApJS, 78, 517

Straniero O., \& Chieffi A. 1991, ApJS, 76, 525 